\title{
El abandono en las carreras en Ciencias Biológicas de la Universidad Nacional de Tucumán. Década 1991-2001
}

\author{
Mariela Maya, Rosana González, Gabriela Aguirre, Jorgelina Juárez, Yanina Fernández y \\ Virginia Abdala
}

\section{RESUMEN}

En este trabajo se presenta una aproximación a la identificación de los aspectos asociados con el abandono de las carreras del profesorado y licenciatura en Ciencias Biológicas durante el periodo 1991-2001 en la Facultad de Ciencias Naturales de la Universidad Nacional de Tucumán, Argentina. La población de estudio estuvo constituida por los alumnos inscritos en dichas carreras durante los años 1990 y 1991 (licenciatura) y durante 1996 (profesorado). Los datos se obtuvieron de las fichas de ingreso de los años citados, se empleó como instrumento la escala Likert para medir actitudes favorables, desfavorables y neutras hacia las carreras. Los resultados indican que los motivos aducidos para el abandono de los estudios se relacionan con causas personales $(73 \%)$, un $9 \%$ adujo problemas económicos y un $5 \%$ problemas de discriminación. Es muy interesante que nuestros datos indican que el porcentaje de estudiantes que aduce causas personales, muestra un registro de rendimiento académico muy irregular.

Palabras clave: biografia, profesor, matemática, escuela secundaria, Argentina.

Mariela Maya Argentina, Especialista en Educación Universitaria, Universidad Tecnológica Nacional, Argentina, Licenciada en Ciencias Biológicas, Facultad de Ciencias Naturales (FCN) e IML, Universidad Nacional de Tucumán (UNT), Argentina. Jefe de Trabajos Prácticos de la Cátedra de Biología General y Metodología de la Ciencia, FCN e IML, UNT, Argentina.

\section{Rosana González}

Argentina. Auxiliar graduada de la Cátedra de Biología General y Metodología de la Ciencia y de Didáctica General, FCN e IML, Universidad Nacional de Tucumán, Argentina. Profesora en Ciencias Biológicas, FCN e IML, UNT, Argentina.

\section{Gabriela Aguirre}

Argentina. Doctora en Arqueología, Universidad Nacional de la Plata. Jefe de Trabajos Prácticos de la Cátedra de Biología General y Metodología de la Ciencia y de Bioarqueología, Facultad de Ciencias Naturales e IML, Universidad Nacional de Tucumán, Argentina.

\section{Jorgelina Juárez}

Argentina, Doctora en Ciencias Biológicas, Universidad Nacional de Tucumán. Jefe de Trabajos Prácticos de la Cátedra de Biología General y Metodología de la Ciencia, FCN e IML, UNT, Argentina. 


\section{O abandono nos cursos de Ciências Biológicas da Universidade Nacional de Tucumán. Década 1991-2001}

\section{RESUMO}

Neste trabalho se apresenta uma aproximação à identificação dos aspectos associados com o abandono dos cursos de licenciatura e bacharelado em Ciências Biológicas durante o período 1991-2001 na Faculdade de Ciências Naturais da Universidade Nacional de Tucumán, Argentina. A população de estudo esteve constituída pelos alunos inscritos em ditos cursos durante os anos de 1990 e 1991 (bacharelado) e durante o ano 1996 (licenciatura). Os dados se obtiveram das fichas de inscrição dos anos citados, se empregou como instrumento a escala Likert para medir atitudes favoráveis, desfavoráveis e neutras sobre os cursos. Os resultados indicam que os motivos prováveis para o abandono dos estudos se relacionam com causas pessoais (73\%), 9\% mencionou problemas económicos e 5\% problemas de discriminação. É muito interessante que nossos dados indicam que a porcentagem de estudantes que mencionou causas pessoais, mostram um registro de rendimento académico muito irregular.

Palavras chave: biologia, educação, abandono, universidade, Argentina.

\section{School Dropout in the Careers in Biological Sciences of the National University of Tucumán. Decade 1991-2001}

\section{ABSTRACT}

This paper presents an approach to the identification of aspects associated with the school dropout of the careers of teachers and bachelors in Biological Sciences during the period 1991-2001 in the Faculty of Natural Sciences of the National University of Tucumán, Argentina. The study population consisted of students enrolled in these careers during the years 1990 and 1991 (bachelor's degree) and during the year 1996 (faculty). The Likert scale was used as an instrument to measure favorable, unfavorable, and neutral attitudes toward careers. The results indicate that the reasons given for dropping out of studies are related to personal causes (73\%), 9\% alleged economic problems and 5\% discrimination problems. Interestingly, our data indicate that the percentage of students who cite personal causes shows a very irregular record of academic performance.

Key words: biology, education, school dropout, university, Argentina.

Recepción: 07/10/16. Aprobación: 18/05/18. 


\section{Introducción}

La universidad argentina modificó considerablemente su situación político-institucional luego de que se sancionara la Ley de Educación Superior (LES), en 1995. Ésta instauró en la educación una profunda reforma que tuvo como principal objetivo la reducción de los "gastos" del Estado en materia educativa y la descentralización de los servicios educativos (Bianchi, 2004). Previamente, en 1993, se firmó un decreto de desregulación de las universidades privadas, que permitía a las universidades crear nuevas carreras, modificar planes de estudio y reformar su estructura organizativa mediante la supresión de los alegados excesivos recaudos del Estado (Ramallo, 2002). Así, la década de los noventa vio incorporarse a las universidades e institutos privados al Sistema Universitario Nacional (artículo 26 de la LES, dentro de la Ley Federal de Educación (LFE)), con autonomía académica e institucional (artículo 29 de Ley de Educación Superior).

En 1996 se dicta el Decreto 173 que establece la integración y funcionamiento de la Comisión Nacional de Evaluación y Acreditación Universitaria (CONEAU) como organismo descentralizado del Ministerio de Gultura y Educación de la Nación. Esta institución está encargada de realizar las evaluaciones externas de las instituciones universitarias y acreditar los posgrados y las carreras profesionales reguladas por el Estado.

Es importante destacar que la Ley Federal de Educación establece una obligatoriedad de nueve años comprendidos en la Educación General Básica (EGB). Esta obligatoriedad se modifica con la nueva Ley de Educación Nacional promulgada en 2003, que establece 12 años de educación obligatoria. Estas innovaciones en la legislación fueron acompañadas con políticas de estado destinadas a asegurar la permanencia y finalización de los estudios preuniversitarios, por ejemplo la Asignación Universal por Hijo o el plan Progresar (Ministerio de Desarrollo Social; http://www.progresar.anses.gob.ar/). Sin embargo, la educación universitaria se mantuvo durante la década del 2000 y hasta la actualidad, regulada por la misma (LES) de 1995.

En el campo educativo, deserción significa abandono temporal o definitivo de la educación formal, es decir, dejar los estudios primarios, secundarios o superiores (Rizzuto, 2009). Tradicionalmente se ha llamado "desertor" al alumno que no completa su formación académica, dejándola por inasistencias, por reprobación o por vencimiento de las materias cursadas (Rizzuto, 2009). Tinto (1982) define a la deserción como una situación a la que se enfrenta un estudiante cuando aspira concluir su proyecto educativo y no logra hacerlo. También se entiende como deserción en la universidad a la interrupción o abandono del proceso académico-institucional que lleva a cabo el estudiante universitario (Tinto, 1989). Una concepción diferente a las mencionadas es la que proponen De Vries et al. (2011), quienes consideran inapropiado el rótulo de desertor para aquellos estudiantes que abandonaron la universidad pero continuaron otros estudios superiores o se insertaron exitosamente en el ámbito laboral. Para este trabajo, y debido a las connotaciones negativas de la palabra desertores, hemos optado por el término abandonadores, siguiendo a Simone y otros (2013). Estos autores definen como abandonadores a los alumnos que se inscribieron en alguna de las carreras, comenzaron el cursado y que al momento del relevamiento no estaban reinscritos, no eran alumnos regulares de la facultad y no se habían graduado. A lo largo de estos años, y a pesar de las continuas modificaciones implementadas en la política educativa nacional, la deserción universitaria se ha mantenido entre el 30 y el 50\% (Domínguez, 2007; Biolatto et al., 2010). La Universidad Nacional de Rosario, por ejemplo, presenta una tasa de abandono de alrededor del 40\% (Biolatto et al., 2010). Así, el ingreso al sistema universitario público puede representarse gráficamente como una curva creciente y el egreso como una recta plana entre los años 1982 y 2000 (Nicoletti, 2010). 
Entre las causas que se aducen para explicar esta abrumadora tasa de deserción se encuentra el "pasar del mundo protegido de la escuela al desamparo de las universidades, donde cada uno debe arreglárselas como pueda" (Follari, 2005). Asimismo se plantean cuestiones económicas, falta de trabajo, disponibilidad horaria que acarrea el trabajar, incertidumbre laboral, etcétera (Panaia, 2013; Chiechier, 2013; Simone et al., 2013). Del análisis de la deserción y del rendimiento académico de los alumnos de la Universidad Nacional de Tres de Febrero (UNTREF), Lorenzano (2004) concluye que los que abandonaron, de entre 22 y 34 años, tienen en su mayoría un trabajo permanente $(32 \%)$, dependen económicamente de ese trabajo $(36 \%)$ y son de sexo masculino $(38 \%)$. En concordancia con estos datos, Rizzuto (2009) determinó como resultados relevantes de su investigación acerca de la deserción, que un gran porcentaje $(71 \%)$ de los alumnos encuestados relacionan el abandono con problemas económicos.

$\mathrm{El}$ abandono es aparentemente un problema universal. Entre los países de América Latina, Chile, de acuerdo con estimaciones basadas en sus propias estadísticas, presenta en el año 2004 una tasa de deserción global de pregrado cercana al 53.7\%, siendo mayor en las universidades privadas nuevas que en las públicas, y menor entre las mujeres $(43 \%$ ) que entre los varones (50\%) (Díaz Peralta, 2008). En países supuestamente desarrollados como Estados Unidos, Francia, España y Austria, el abandono oscila entre el 30 y el 50\%. Estas cifras contrastan con las de los países de mejor desempeño como Alemania con un 20\% y Finlandia, con un 10\% (Lewis, 2000; Vélez y López Jiménez, 2004).

La Universidad Nacional de Tucumán presenta una gran oferta académica, entre la cual se encuentran las carreras de profesorado y licenciatura en Ciencias Biológicas, que se dictan en la Facultad de Ciencias Naturales (FCN) e Instituto Miguel Lillo. Los planes de estudios de estas carreras se han modificado en los últimos 20 años. El del profesorado se modificó en 1996. Entre los cambios generados se propone un título disciplinar: Profesor en Ciencias Biológicas, en lugar del título de Profesor en Ciencias Naturales. Posteriormente, en 2000, se modifica nuevamente para lograr una unificación de los contenidos con la licenciatura en Ciencias Biológicas, compartiendo el $80 \%$ de las materias. Por su parte, el plan de estudio de la licenciatura en Ciencias Biológicas también fue objeto de modificaciones. El Plan de Estudios 2000, modificado en 2007, fue generado con base en los lineamientos establecidos por el Consejo Interuniversitario para la Enseñanza Superior de la Biología (CIPEB). En la actualidad se encuentra en análisis una nueva propuesta de plan de estudios en el Ministerio de Educación de la Nación. A pesar de todas estas modificaciones y de otras acciones destinadas a disminuir el abandono en la Facultad, éste se ha mantenido muy alto en las últimas décadas. El seguimiento de la cohorte 2002 hasta el año 2012 muestra que de 165 alumnos que ingresaron a la carrera de licenciatura en Ciencias Biológicas, sólo 11 egresaron $(6.6 \%)$, mientras que la duración de la misma, formalmente de cinco años, en la práctica se extiende a 7.8 años (Maya, 2013). Estos datos coinciden con la Resolución 354/14 emitida por CONEAU en 2014, que muestra una duración real de 8 años.

En este trabajo presentamos una primera aproximación a la identificación de los aspectos más relevantes que podrían asociarse con el abandono de las carreras universitarias del profesorado y licenciatura en Ciencias Biológicas durante el periodo 1991-2001 en nuestra Facultad, respecto del cual se cuenta con datos aproximadamente suficientes. Seleccionamos esta década también por la relevancia de las políticas adoptadas en distintos ámbitos educativos con implicancia sobre la vida social. A mediados de los años ochenta se inicia una serie de reformas que repercutirán en todos los ámbitos de la vida social, con un fuerte impacto durante la década de los noventa (Bianchi, 2004). El citado autor, apoyándose en distintos investigadores considera que: "las 
políticas neoconservadoras han afectado profundamente a la sociedad en general y al sistema educativo en especial, por ser éste parte integrante del entorno social"(Bianchi, 2004: 2). Santos Sharpe (2016) señala que hacia 1984 se produjeron nuevas condiciones institucionales y materiales para el desarrollo de estudios sobre la temática del abandono, ya que se estableció nuevamente el ingreso irrestricto en las universidades y con esto la masificación de las mismas.

Con la sanción de la LES en Argentina, y los debates sobre los modos de financiamiento de las instituciones universitarias durante la misma década de los noventa, se producen trabajos sobre el abandono universitario con relación al financiamiento y graduación y a la relación graduados-mercado de trabajo (Santos Sharpe y Carli, 2016). Esta línea de análisis se ubica dentro del modelo de la teoría del Capital humano, planteada también por García de Fanelli (2005).

Con base en a la literatura analizada (Lorenzano, 2004; Bianchi, 2004; García de Fanelli, 2005; Rizzuto, 2009; Santos Sharpe, 2016) partimos del supuesto de que la principal causa de la deserción de las carreras del profesorado y licenciatura de la FGN durante la década seleccionada se relacionará fundamentalmente con razones socioeconómicas más que académicas o vocacionales.

\section{Metodología \\ Población de estudio}

Trabajamos identificando a los abandonadores que se inscribieron en las carreras de licenciatura en Ciencias Biológicas de la FGN durante 1990 y 1991 y en profesorado en Ciencias Biológicas en 1996. Los años se seleccionaron de modo de incorporar en los planes de estudio anteriores a los vigentes, ya que no existen datos o estudios realizados con respecto a estos planes de estudio. Para la obtención de estos datos se revisaron los registros de inscripciones y calificaciones de ingreso de estos estudiantes durante los años citados, archivadas en Sección Alumnos de la FCN. Estos registros consignan los datos personales de los estudiantes, fechas de inscripción y cursado, regularidad en las materias, las materias aprobadas, regularizadas/libres, desaprobadas (Anexo 1. Registros de inscripciones y calificaciones). Es importante destacar que Sección Alumnos no archiva registros de los estudiantes que no aprobaron materias. A continuación se procedió a la búsqueda de contactos con estos alumnos, para lo cual se apeló a conocimiento personal, redes sociales, correos electrónicos, etcétera. Se destinaron cuatro meses al esfuerzo de búsqueda. Una vez contactados, se envió a cada estudiante la encuesta desarrollada por nuestro equipo, cuya confección se explica posteriormente. De un total de 472 individuos de la carrera de licenciatura y 57 de profesorado que no finalizaron sus estudios, se identificaron 53, de los cuales 22 contestaron las encuestas completas, uno se negó y los restantes no pudieron ser encuestados (cambio de domicilio, cambio de teléfono, etcétera). Se realizó un análisis detallado de los registros de los estudiantes que contestaron las encuestas a fin de profundizar en los motivos de su abandono. El primer análisis comprende el número de materias aprobadas por año, el segundo el porcentaje de materias aprobadas según el plan de estudios correspondiente. Dado que los estudiantes encuestados corresponden a diferentes carreras y diferentes planes de estudio, se agruparon en cuatro conjuntos: profesorado en Ciencias Naturales (Plan 1964), profesorado en Ciencias Biológicas (Plan 93/94), licenciatura en Ciencias Biológicas Or. Zoología (Plan 1987) y Licenciatura en Ciencias Biológicas Or. Botánica (Plan 1987) (Anexo 2. Materias de cada plan).

Los datos se trataron según la formula $([R]$ 91$[\mathrm{I}+\mathrm{R}]$ 90=I - $\mathrm{R}-\mathrm{E}=$ ), donde $\mathrm{R}$ corresponde a alumnos reinscritos por año, I corresponde a nuevos inscritos por año y E corresponde a número de egresados por año (Anexo 3. Guadro mostrando el índice de abandono; www.herrera.unt.edu.ar/deunt/ desercion/desercion.htm). 


\section{Instrumento}

Como instrumento básico se implementó la escala Likert, utilizada frecuentemente en el estudio de las actitudes sociales, con el propósito de medir actitudes favorables, desfavorables y neutras hacia la carrera. Esta es una escala ordinal y como tal no mide en cuánto es más favorable o desfavorable una actitud. A pesar de esta limitación, la escala Likert tiene la ventaja de que es fácil de construir y de aplicar; además, proporciona una buena base para una primera ordenación de los individuos en la característica que se mide (Fernández de Pinedo, 1982). La misma fue modificada para adecuarse a nuestra investigación. Se obtuvieron los datos personales de los estudiantes y las razones de abandono formuladas en tres categorías: causas personales, académicas y socioeconómicas.

Las afirmaciones destinadas a identificar las causas personales fueron:

- Elegí la carrera por vocación

- Dejé los estudios por problemas personales

- Dejé los estudios por maternidad o paternidad

- Dejé los estudios por problemas de salud

- Dejé los estudios por problemas de adaptación a la vida universitaria

- Dejé los estudios por falta de vocación.

Las afirmaciones destinadas a identificar las causas socioeconómicas fueron:

- Dejé los estudios por problemas económicos

- Dejélos estudiosporquemesentídiscriminado/a.

Estas afirmaciones podían responderse de acuerdo con una escala (Anexo 3). Las afirmaciones destinadas a identificar las causas académicas fueron:

- El plan de la carrera me pareció exigente

- Las exigencias académicas fueron demasiadas

- El régimen de asistencia fue muy rígido
- Los exámenes parciales y/o finales me parecieron difíciles

- Los contenidos de las materias me parecieron demasiado exigentes

- Dejé los estudios para continuar y finalizar otra carrera de educación superior

- La infraestructura para el dictado de clases me pareció insuficiente

- Dejé los estudios porque la carrera no era lo que pensaba

- Sentí compromiso por parte de los docentes

- Mis inquietudes fueron respondidas a tiempo por los docentes.

\section{Evaluación de los ítems}

Para el análisis de los datos se utiliza el modelo de estadística descriptiva, teniendo en cuenta variables cuasi cuantitativas u ordinales. A cada valor de respuesta se le asignó un puntaje teniendo en cuenta si los ítems son positivos o negativos. Si el enunciado es positivo, se asigna el valor de 5 a Muy de acuerdo, hasta llegar a 1, Muy en desacuerdo. Si el ítem es negativo, se le asigna 1 a Muy de acuerdo, hasta llegar a 5 a Muy en desacuerdo. Por cada persona que responde, se suma el resultado obtenido y se divide por la cantidad de preguntas, en este caso 19. En nuestro caso: puntaje máximo por obtener: 95/ 19 ítems $=5$; puntaje mínimo por obtener: 19/ 19 ítems $=1$.

De acuerdo con lo anterior, la actitud de los encuestados se evalúa como: 5 : muy positiva; 4 : positiva; 3: neutra; 2: negativa, y 1: muy negativa (Anexo 3. Análisis de respuestas por ítems).

\section{Resultados}

A continuación, teniendo en cuenta las causas analizadas: personales, académicas y socio-económicas, se presentan en primer lugar la descripción general de los resultados (tabla 1), posteriormente se corrobora la confiabilidad de la escala y finalmente se realiza el análisis por dimensiones de las variables. 
De los 22 encuestados, teniendo en cuenta la valoración hacia la carrera aportada por la escala Likert (Anexo 4. Valoración escala Likert; tabla 1), 14 respondieron de manera positiva $(64 \%)$ y 8 de manera neutra (36\%) (figura 1). Entre los neutros, el 75\% corresponde a mujeres y el resto a varones (figura 2).

Para medir la confiabilidad de la escala, se calculó el coeficiente alpha de Cronbach en Excel mediante la varianza de los ítems, cuyo valor fue de 0.57 , lo que significa una confiabilidad moderada.

Tabla 1. Resultados generales

\begin{tabular}{|l|c|}
\hline Casos & 22 \\
\hline İtems & 19 \\
\hline Media & 67.87 \\
\hline DS & 8.57 \\
\hline Mediana & 68 \\
\hline Porcentaje Mínimo & 56 \\
\hline Porcentaje Máximo & 82 \\
\hline
\end{tabular}

Figura 1. Porcentaje de encuestados con actitud positiva o neutra hacia la carrera

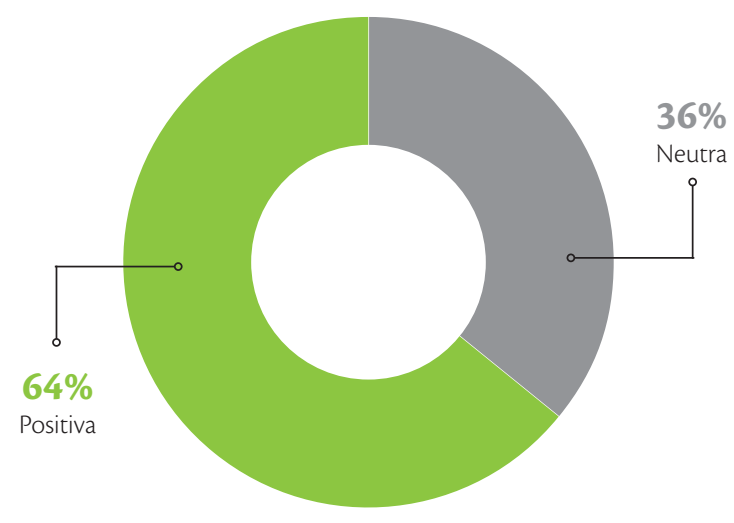

Figura 2. Causas de abandono discriminadas por género

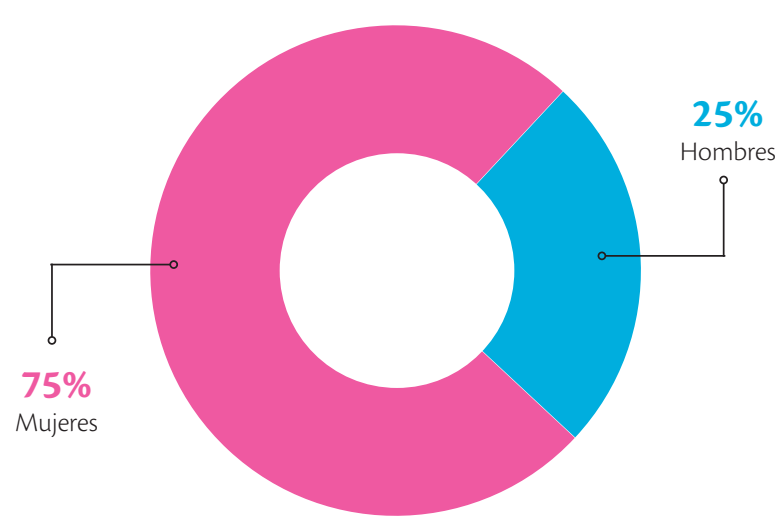


El 62.5\% de las mujeres abandona la carrera por causas relacionadas con maternidad, salud y económicas, mientras que sólo una encuestada aduce problemas académicos. Entre los varones, el 50\% abandona por causas académicas y el $50 \%$ por paternidad (figura 3).

De las afirmaciones destinadas a identificar las causas personales destacan que:

- En el ítem 2, el 14\% no eligió la carrera por vocación.

- En el ítem 11, el 73\% abandonó la carrera por problemas personales.

- En el ítem 12, el 32\% dejó los estudios por maternidad o paternidad.

- En el ítem 13, el 14\% abandonó por problemas de salud.

- En los ítems 16 y 17, el 5\% dejó por falta de adaptación a la vida universitaria y de vocación, respectivamente.

- En el ítem 18, el 5\% dejó la carrera porque no era lo que pensaba y en el ítem 19, el 36\% dejó la carrera para continuar y finalizar otra carrera de nivel superior (figura 4).

De los ítems destinados a identificar las causas académicas:

- En el ítem 1, el 42\% cursó previamente otra carrera de nivel superior.

- En el ítem 3, el 9\% piensa que el plan de estudio fue exigente.

- En los ítems, 4, 5 y 6 el 14\% está de acuerdo con que las exigencias académicas, de contenidos y el régimen de asistencia respectivamente fueron elevadas.

- En los ítems 7 y 8 , el $18 \%$ respondió que los exámenes fueron difíciles y la infraestructura insuficiente, respectivamente.

- En el ítem 9 sólo el 5\% sintió la falta de compromiso por parte de los docentes, y en el ítem 10 el $9 \%$ manifestó que sus inquietudes no fueron respondidas a tiempo por los docentes (figura 5).

Figura 3. Porcentaje de encuestados según su género

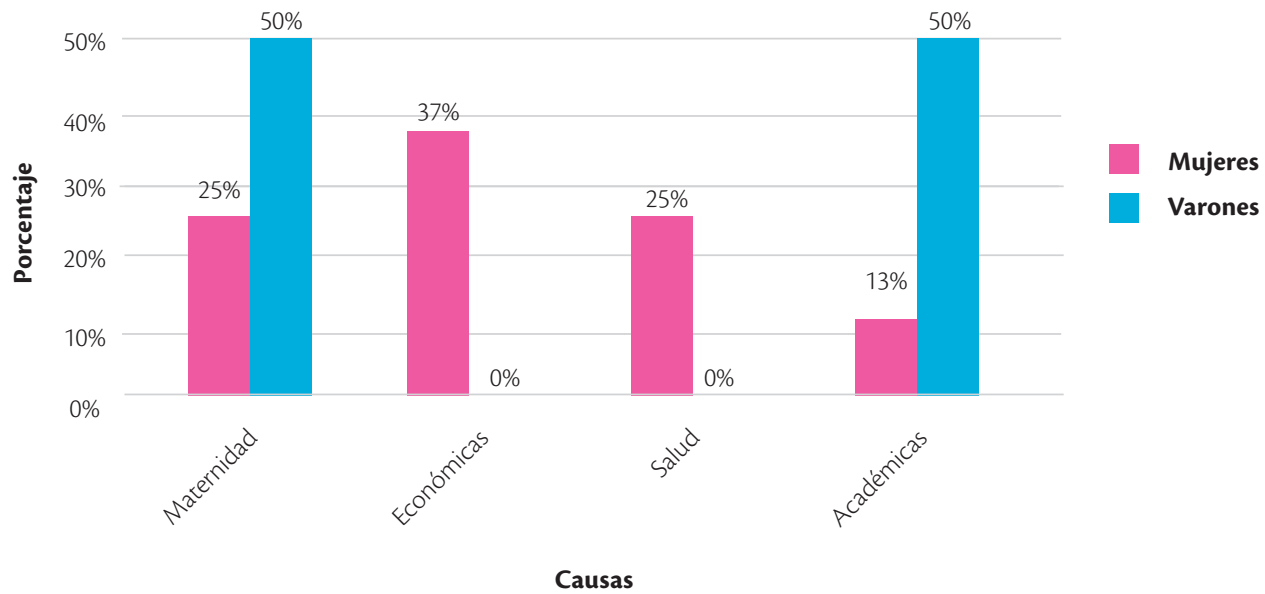


De los ítems destinados a reconocer las causas socioeconómicas (figura 6) destacamos que: en el ítem 14, el 9\% abandonó la carrera por problemas económicos, en tanto que en el ítem 15, sólo el 5\% dejó por problemas de discriminación. El año de mayor abandono es el $3^{\circ}$ año (50\%), seguido por el $2^{\circ}(23$ \%) (Anexo 5. Resultados año de mayor abandono; figura 7).

Figura 4. Afirmaciones referidas a causas personales

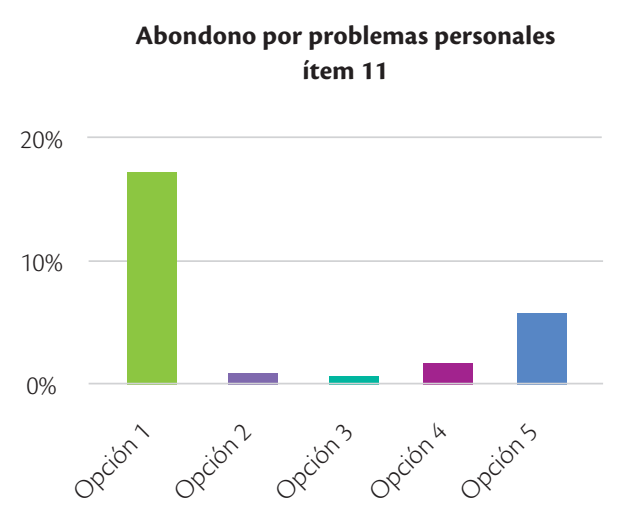

Abandono por maternidad / paternidad ítem 12

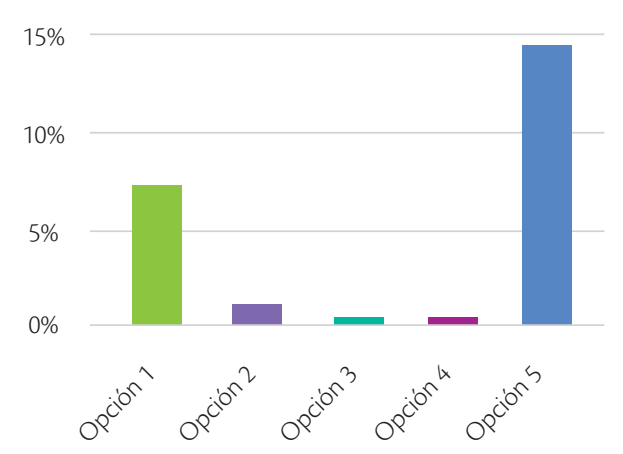

Abandono por discriminación ítem 15

$30 \%$

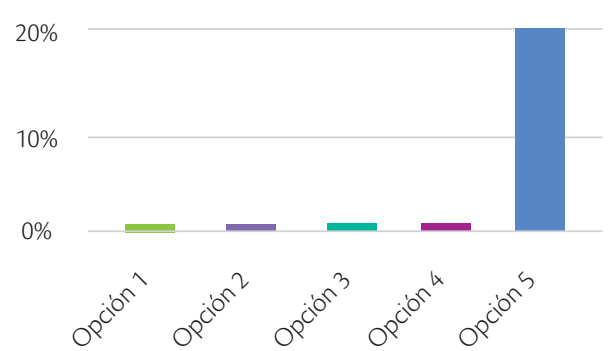

Elección de la carrera por vocación ítem 2

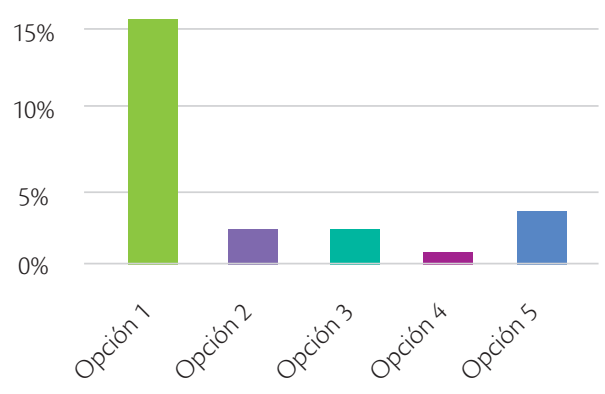

Abandono por problemas de salud ítem 13

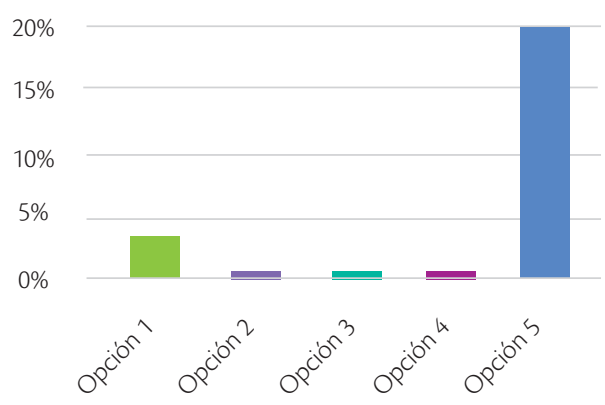

Abandono por falta de vocación ítem 17

$30 \%$

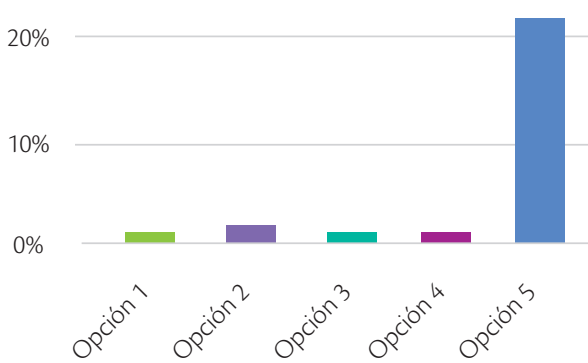


Abandono por desilución por la carrera ítem 18

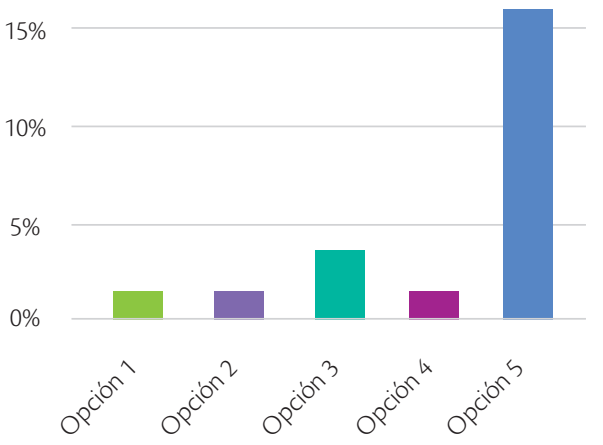

Abandono para realizar otra carrera ítem 19

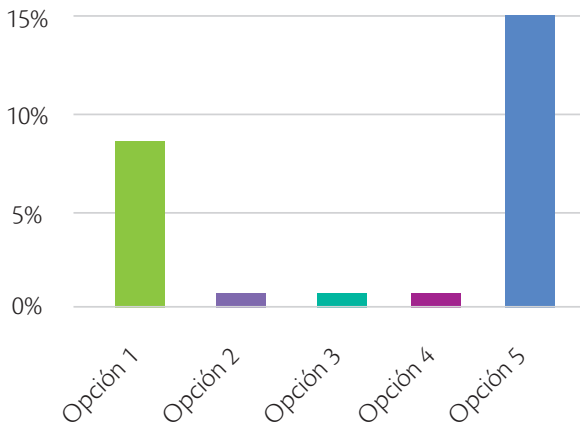

Figura 5. Afirmaciones referidas a causas académicas

Exigencia plan de estudio ítem 3

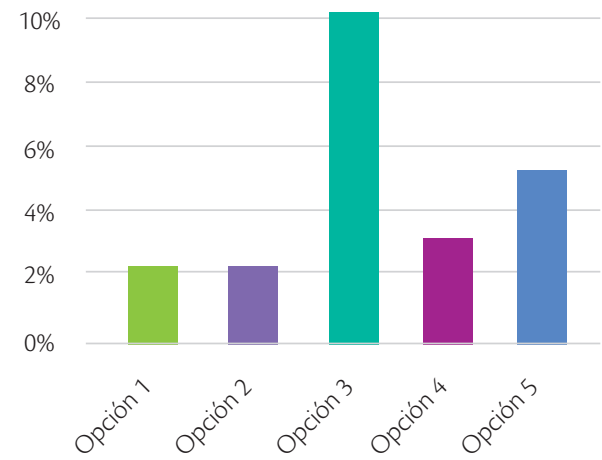

Exigencias de los contenidos ítem 5

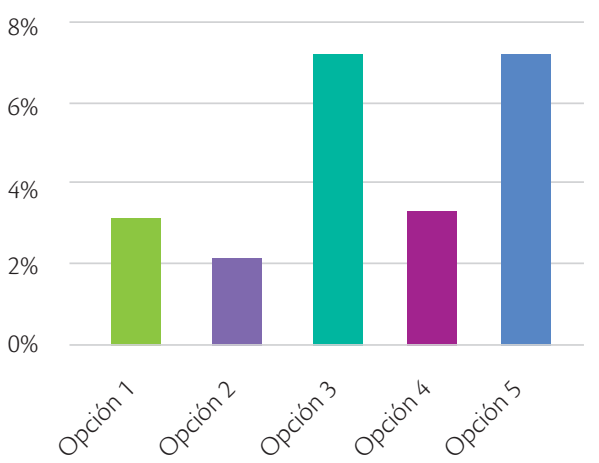

Exigencias académicas ítem 4

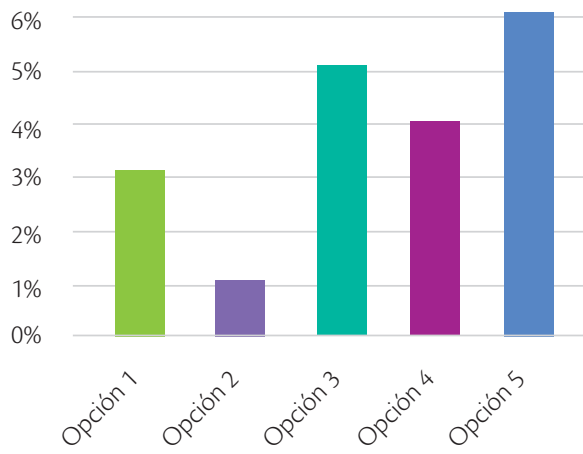

Régimen de asistencia ítem 6

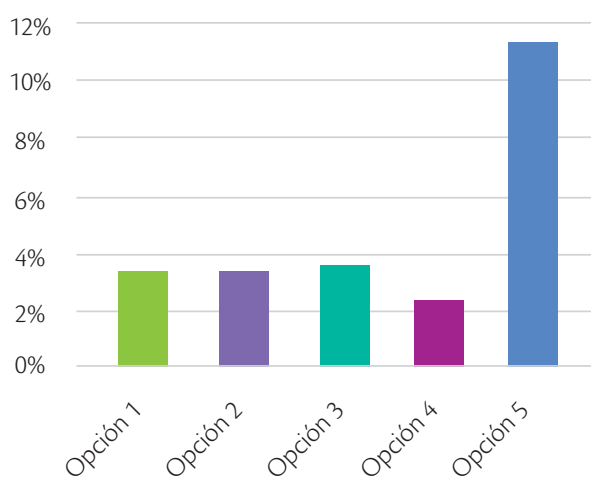




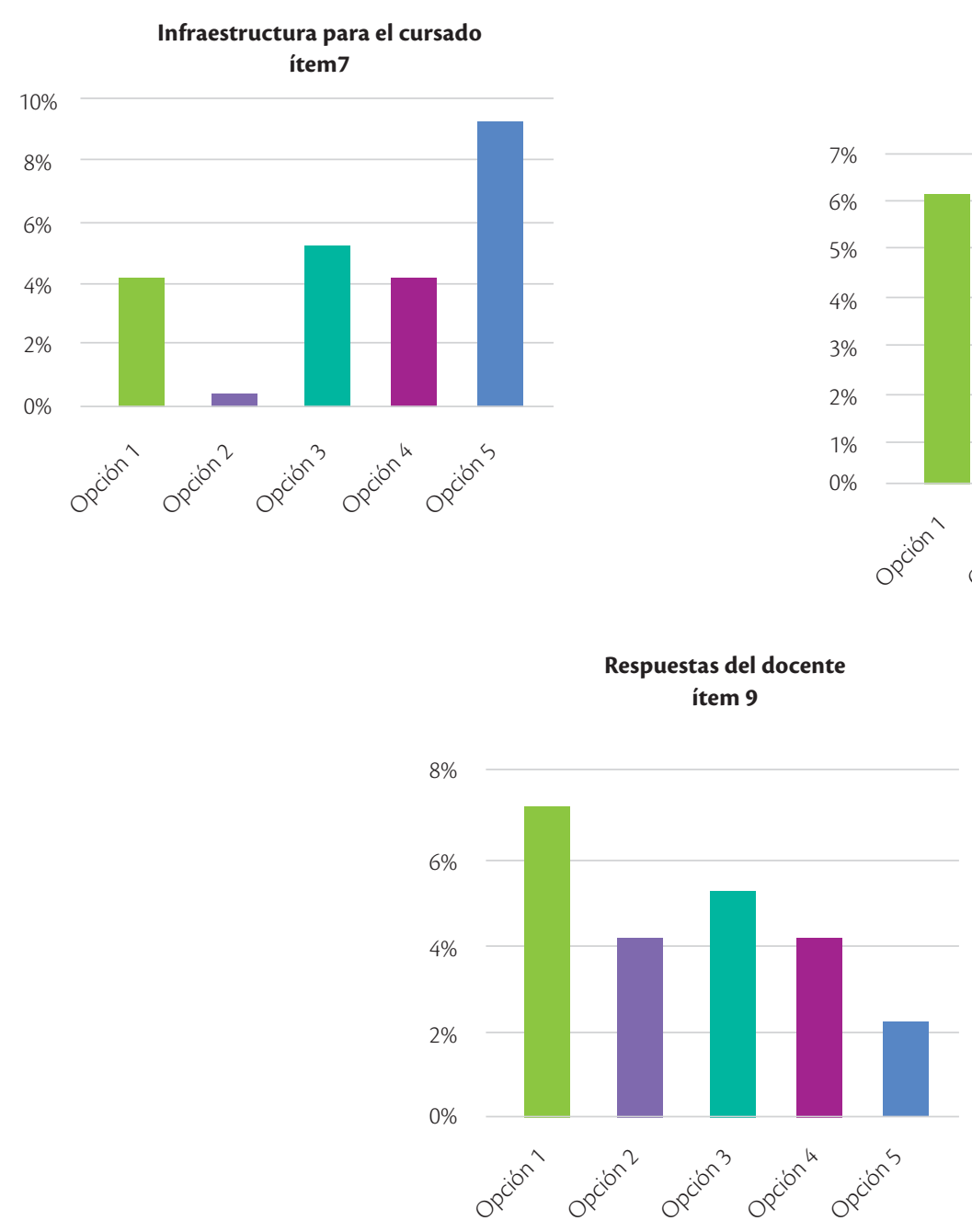

Compromiso docente

ítem 8

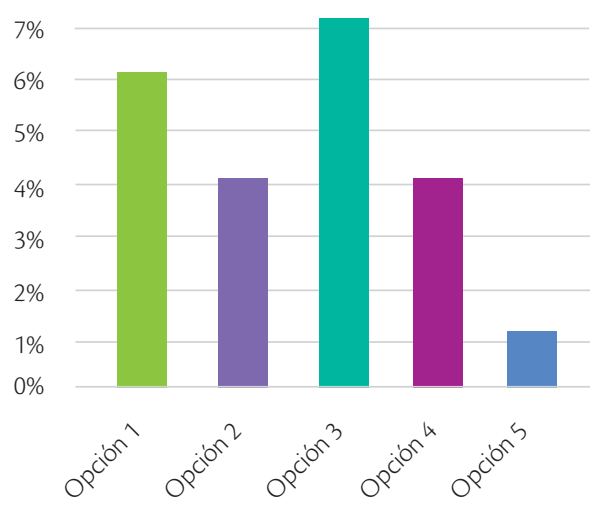

Figura 6. Afirmaciones referidas a causas socioeconómicas

Abandono por problemas económicos ítem 114
Abandono por discriminación ítem 15

$30 \%$
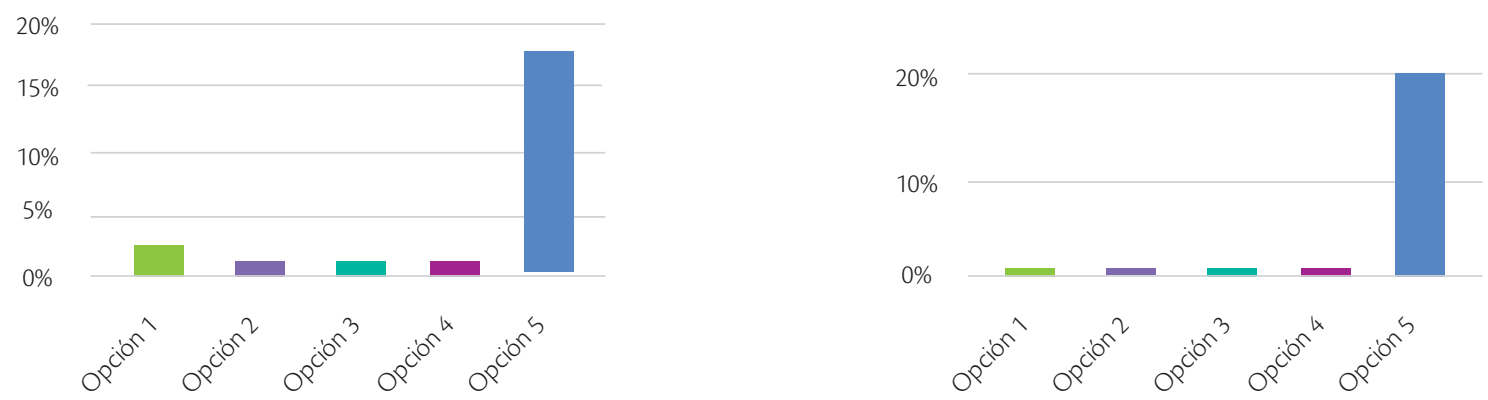
Figura 7. Porcentaje de abandono de la carrera según año de cursado

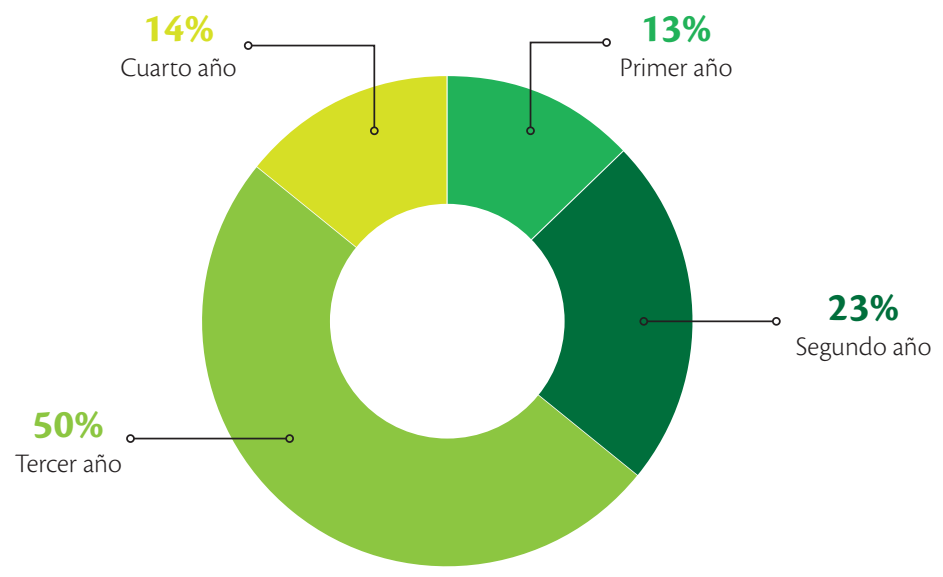

Con respecto al análisis detallado de los registros de los cuatro grupos, ver figuras $8,9,10$ y 11 :
Las respuestas de los encuestados en relación con las causas de su abandono y los datos de su desempeño académico se observan en la tabla 2.

Figura 8. Número de materias aprobadas por año

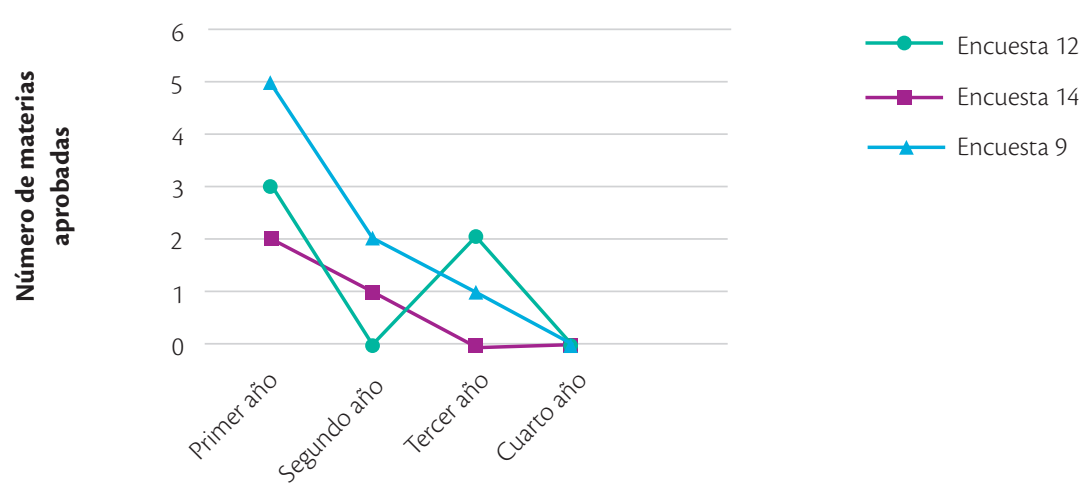

Años de carrera 
Figura 9. Número de materias aprobadas por año

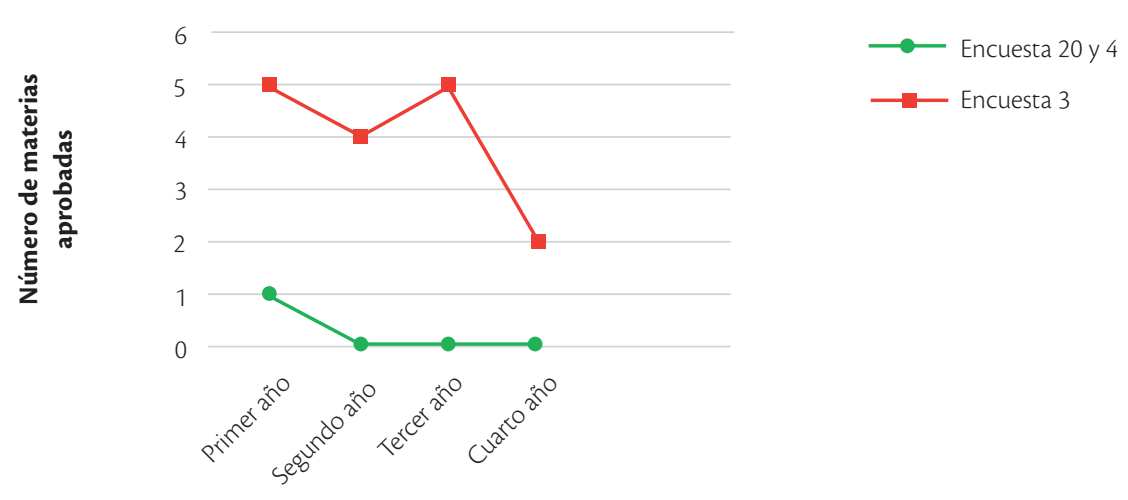

Años de carrera

Figura 10. Número de materias aprobadas por año

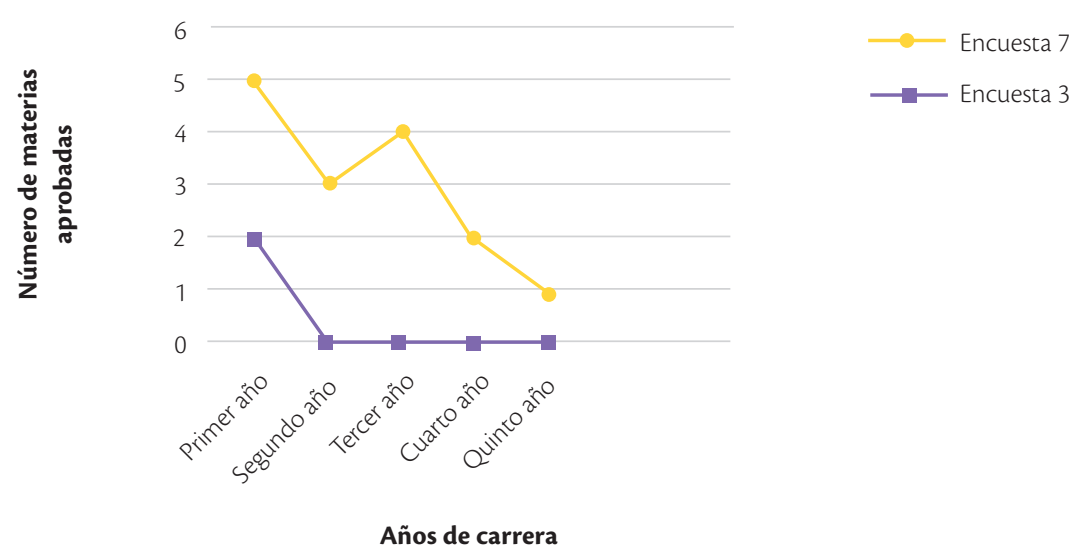

Figura 11. Número de materias aprobadas por año

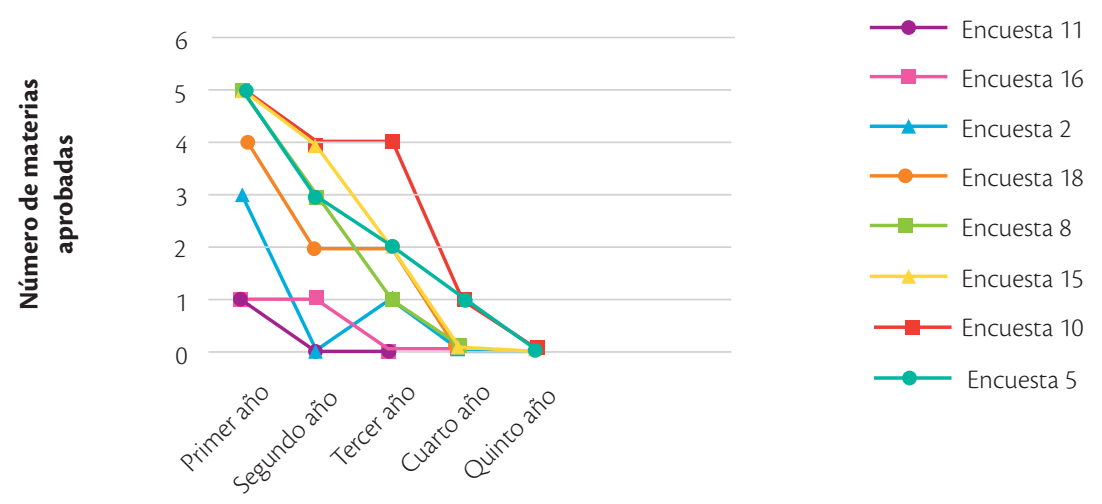

Años de carrera 
Tabla 2. Desempeño académico

\begin{tabular}{|c|c|c|c|c|c|c|}
\hline Encuestados & $\begin{array}{c}\text { Porcentaje } \\
\text { de materias } \\
\text { aprobadas en } \\
1^{\circ} \text { año }\end{array}$ & $\begin{array}{c}\text { Porcentaje } \\
\text { de materias } \\
\text { aprobadas en } \\
2^{\circ} \text { año }\end{array}$ & $\begin{array}{c}\text { Porcentaje } \\
\text { de materias } \\
\text { aprobadas en } \\
3^{\circ} \text { año }\end{array}$ & $\begin{array}{c}\text { Porcentaje } \\
\text { de materias } \\
\text { aprobadas en } \\
4^{\circ} \text { año }\end{array}$ & $\begin{array}{c}\text { Porcentaje } \\
\text { de materias } \\
\text { aprobadas en } \\
5^{\circ} \text { año }\end{array}$ & $\begin{array}{c}\text { Causa de abandono según encuesta } \\
\text { personal }\end{array}$ \\
\hline 20 & 20 & 0 & 0 & 0 & - & Personales/Dejó para continuar otra carrera \\
\hline 4 & 20 & 0 & 0 & 0 & - & Personales \\
\hline 3 & 100 & 100 & 100 & 33 & - & Personales/maternidad \\
\hline 12 & 50 & 0 & 40 & 0 & - & Personales/ Dejó para continuar otra carrera \\
\hline 14 & 33 & 0 & 20 & 0 & - & La carrera no era lo que pensaba \\
\hline 9 & 83 & 33 & 20 & 0 & - & Problemas de salud \\
\hline 7 & 100 & 60 & 80 & 40 & 20 & Salud/económico \\
\hline 6 & 20 & 0 & 0 & 0 & 0 & Personales/falta adaptación \\
\hline 2 & 20 & 0 & 0 & 0 & 0 & Problemas personales \\
\hline 5 & 100 & 60 & 20 & 20 & 0 & Problemas personales \\
\hline 8 & 100 & 60 & 20 & 0 & 0 & Problemas personales/maternidad \\
\hline 10 & 100 & 80 & 80 & 20 & 0 & Problemas personales/maternidad \\
\hline 11 & 60 & 0 & 20 & 0 & 0 & Problemas personales/paternidad \\
\hline 18 & 80 & 40 & 60 & 0 & 0 & Problemas personales \\
\hline 15 & 100 & 80 & 40 & 0 & 0 & Dejó la carrera para continuar otra \\
\hline 16 & 20 & 20 & 0 & 0 & 0 & $\begin{array}{c}\text { Falta de vocación/la carrera no era la } \\
\text { esperada }\end{array}$ \\
\hline
\end{tabular}




\section{Discusión y conclusiones}

El supuesto inicial para este trabajo fue que el abandono durante la década seleccionada se relacionaría fundamentalmente con razones socioeconómicas más que con las académicas o vocacionales. Si bien el número de encuestados no es representativo en términos del universo en estudio, por lo que no nos permite realizar generalizaciones en todos los casos, sí podría delimitar un escenario que nos ayude a considerar ciertos aspectos que generarían el abandono.

El resultado alcanzado del coeficiente alfa de Cronbach (0.57) muestra una confiabilidad moderada. Este valor es aceptable en las primeras fases de una investigación siguiendo a Nunnally (1967), que considera que un valor de fiabilidad de 0.6 o 0.5 puede ser suficiente. Para analizar los resultados de una manera más detallada a partir de la medición de este coeficiente, se debe tener en cuenta: la homogeneidad del grupo, el tiempo, el tamaño del cuestionario y la objetividad del proceso de asignar puntuaciones.

$\mathrm{El}$ análisis de las encuestas muestra que los motivos más aducidos para el abandono de los estudios de Biología en nuestra Facultad se relacionan con causas personales. Un 86\% de los encuestados afirmó haber elegido la carrera por vocación y un 73\% haberla abandonado por problemas personales. Siguiendo a Chiecher (2013), se define como causas personales a aquellos acontecimientos vitales particulares de los sujetos, que interfieren negativamente en sus trayectorias de estudio.

Un 9\% adujo problemas económicos y un 5\% problemas de discriminación. En el plano académico, $14 \%$ adujo exigencias y contenidos académicos elevados y 18\% exámenes difíciles y problemas de infraestructura.

Aunque preliminares, estos resultados son contrastantes con los estudios de Rizzutto (2009) y Lorenzano (2004). Nuestros datos tienden a reforzar lo planteado por Troncoso y Ávila (2007) (Biolatto et al., 2010) para el caso de la Universidad Nacional del Comahue (UNCo), en la cual muchos jóvenes provenientes de diferentes colegios con diversas modalidades, sin apremios económicos, sin problemas cognitivos, deciden abandonar los estudios por razones muy variadas que no necesariamente se explican por motivos laborales o socioeconómicos. Obviamente, los aspectos socioculturales de la problemática analizada no pueden ignorarse, pero tampoco aducirse como la única o más importante causa del abandono. Siguiendo a Charlot (2007) es posible afirmar que reducir la causa del fracaso escolar únicamente al origen social implica cometer por lo menos dos errores: por un lado, pasar variables construidas por el investigador (las posiciones) como realidades empíricas (llamadas origen o fracaso escolar) y por otro lado, interpretar un lazo también construido (la correlación) en términos de causa efectiva, de acción empírica. De acuerdo con lo planteado por Charlot (2007), el fracaso escolar está relacionado con el origen social (si no, no existiría correlación entre dos variables), pero el origen social no produce el fracaso escolar.

Nuestros resultados muestran que la mayor parte de los estudiantes abandonan sus estudios en $3^{\circ}$ año, lo cual se correspondería a la categoría planteada por Fontalvo Cerpa et al. (2014) de deserción tardía, en la cual el individuo abandona los estudios en los últimos semestres. Sin embargo, el análisis de los registros estudiantiles muestra que muchos de ellos no completaron el primer año de la carrera, lo cual sustenta el planteo de Montes (2002) acerca de que los primeros cuatrimestres son críticos. En nuestra experiencia de décadas como docentes en el primer año de nuestra Facultad, hemos notado en los estudiantes la abrumadora sensación de entrar a un mundo cuyos códigos se ignoran y en el que están condenados al más crudo anonimato. La masividad tan característica de los primeros años de casi todas las facultades, incluida la nuestra, ha reemplazado las relaciones personales de una institución que hasta hace alrededor de 30 años recibía menos de 30 ingresantes a la carrera de Biología por año. Esta temática 
ha sido analizada también por Montes (2002), quien plantea que durante el primer semestre, el estudiante está obligado a abandonar la burbuja de la educación media y moverse en la universidad, en la cual debe valerse por sí mismo, tanto en el aula como en los distintos espacios institucionales.

Este momento de mayor abandono coincide con el tercer periodo crítico de abandono entre los identificados por Tinto (1989), quien sugiere que el primer periodo corresponde a la etapa en la que el alumno se contacta con la universidad por los trámites de admisión, la segunda etapa crítica corresponde a la transición entre la enseñanza media y la universidad, luego del ingreso, especialmente en la última fase del primer año de estudios, antes del comienzo del segundo año.

Creemos, como Chiecher (2013), que si bien se puede localizar un momento temporal en el que el sujeto abandona una carrera, la decisión de dejar los estudios va más allá de ese momento concreto y transcurre, más bien, como un proceso que incluye etapas. Según Elbaum (1998), en Jewsbury y Haefeli (2000), ningún abandono se produce por un quiebre instantáneo, sino que es una conflictividad prolongada en el espacio y en el tiempo. El que abandona primero suele sentirse abandonado por la institución. Esto es, más que un evento, la decisión de abandonar es un proceso. Las explicaciones de Montes (2002), Follari (2005), Troncoso y Ávila (2007), Aguilera de Fretes y Chávez (2012) hacen hincapié en el problema de la transición secundario-universidad y los problemas de ajuste que ésta genera. Un examen más detallado de nuestros resultados revela que el $58 \%$ de las mujeres y el $30 \%$ de los varones siguió otra carrera afín pero de nivel terciario. Estos porcentajes estarían señalando dificultades probablemente relacionadas con las exigencias académicas de la carrera. Llamativamente, esto no se desprende de las respuestas de los encuestados. La escala Likert aplicada muestra que el $64 \%$ exhibe una actitud positiva hacia la carrera y un $36 \%$ una actitud neutra, mientras que ninguno exhibe actitudes negativas. Un análisis de las respuestas de los encuestados que exhiben una actitud neutra nos permite profundizar en algunas hipótesis acerca de los motivos de abandono. Observamos respuestas que se diferencian según género; así, la mayoría de las mujeres abandona por causas relacionadas a maternidad, salud y problemas económicos. En general, estas situaciones no eran consideradas en la normativa institucional. En 2013 se aprueba en nuestra facultad una reglamentación $(1008 / 10)$ que contempla las situaciones de cursado de las estudiantes embarazadas, sería interesante replicar nuestro estudio para las próximas décadas y monitorear así la influencia de estas políticas institucionales positivas en la tasa de abandono, además de plantearse estudios que profundicen la problemática de género/abandono en nuestra institución.

Un aspecto que no puede faltar en el análisis de las causas del abandono de cualquier carrera es el relacionado con la valoración del título que se persigue. Esto se refiere al valor que le dan al diploma los grupos de nuevos profesionales y al grado de expectativas que éstos cumplen: nivel de salarios, prestigio social, posibilidad de acceder a cargos de poder, etcétera (Panaia, 2013). Datos disponibles muestran diferencias en las tasas de abandono entre el profesorado y la licenciatura en Ciencias Biológicas (por ejemplo, entre 1996 y 1997 egresaron de licenciatura $41 \%$ de los ingresantes y el 19\% del profesorado). El título de profesor de Biología habilita, obviamente, para la docencia en Biología en el nivel secundario y superior. Los profesores egresados de la FCN rara vez permanecen en la Facultad como docentes y su tarea suele ser subestimada en el contexto institucional. Por el contrario, la carrera de licenciatura está ligada principalmente a la investigación científica, muy prestigiada en nuestra sociedad. Creemos que en el transcurso de la vida social de los individuos que transitan por las carreras mencionadas en la FGN se ha ido alojando un habitus secundario (Bordieau, 1998), producido por la institución formal que 
inculca imperceptiblemente su cultura. Esto es, un habitus, una "subjetividad socializada" que se asienta sobre una subestimación de la carrera del profesorado y sus actores. Si bien no existen estudios al respecto, estos aspectos que se refieren a la construcción del imaginario colectivo en las instituciones merecerían ser evaluados más rigurosamente.

Otro faceta a considerar es la que señala Tinto (2006-2007) acerca del cambio de paradigma en relación con el abandono estudiantil. Hasta la década de los sesenta, las visiones sobre el estudiante que dejaba sus estudios correspondían a la de un individuo menos capaz, menos motivado y que no aprovechaba los beneficios de recibir educación universitaria. Posteriormente se pasó a una mirada que considera la importancia de la relación entre el estudiante y la sociedad, el ambiente y el rol de la institución (Tinto 2006-2007). Es muy interesante que nuestros datos indican que el porcentaje de estudiantes que aduce causas personales, muestra un registro de rendimiento académico muy irregular. Es importante destacar que en las instituciones universitarias no se realiza un análisis suficientemente riguroso de las condiciones de apropiación de los saberes impartidos (Charlot, 2007). Debido a esto, el problema sigue estando en el estudiante, porque se considera que el éxito escolar es asunto sólo de capital personal y no de trabajo, más precisamente, de actividades y prácticas (Charlot 2007).
De esto podría desprenderse que causas que el estudiante asume como un problema personal pueden en realidad estar enmascarando deficiencias institucionales. Por esto, el cambio de paradigma al cual alude Tinto (2006-2007) sigue estando en el plano de lo teórico ya que los estudiantes asumen que son ellos los que no tienen las habilidades intelectuales que hacen falta para concluir su carrera universitaria.

Así, una de las conclusiones más importantes de nuestro trabajo es que la principal causa de abandono aducida por los encuestados está relacionada con causas personales. Sin embargo, un análisis más detallado de los casos revela que detrás de esas causas personales existen trayectorias curriculares muy irregulares que parecen estar señalando deficiencias institucionales que los estudiantes asumen como propias. Por lo que consideramos que las instituciones fallan doblemente: al no ofrecer trayectos curriculares realizables y al no asumir la responsabilidad que estas fallas generan.

\section{Agradecimientos}

Al personal de Departamento Alumnos de la Facultad de Ciencias Naturales e IML de la Universidad Nacional de Tucumán, Lucia Gil, Graciela Díaz y Graciela López, quienes generosamente nos facilitaron información sin la cual no hubiéramos podido concretar este trabajo y nos permitieron el acceso a los datos archivados en esa dependencia. 


\section{Referencias}

Aguilera de Fretes, M. N. y V. E. Jiménez Chavez (2012), "Factores de deserción universitaria en el primer curso de las carreras de Trabajo Social y Lengua Inglesa en las Facultades de Humanidades y Ciencias de la Educación y de Lenguas Vivas de la Universidad Evangélica del Paraguay", en Revista Internacional de Investigaciones en Ciencias Sociales, 8 (2), 197-205.

Bianchi Bustos, M. (2004), "Argentina: la educación desde los '90 a la actualidad", disponible en: <http:/ / firgoa.usc.es/drupal/node/12976>.

Biolatto, R., L. Boccardo y M. C. Lesquiuta (2010), "Acceso y permanencia en una educación de calidad. El ingreso a la universidad, un puente a atravesar", en Congreso Iberoamericano de Educación. Metas 2021, Buenos Aires, Argentina, disponible en: <http://www.adeepra.org. ar/congresos/Congreso\%20IBEROAMERICANO/ ACCESO/R1045_Biolatto.pdf>.

Bordieu, P. (1998), La distinción. Criterios y bases sociales del gusto, Buenos Aires, Taurus.

Charlot, B. (2007), La relación con el saber. Elementos para una teoría, Buenos Aires, Libros del Zorzal.

Chiecher, A. (2013), "Abandonar ingeniería en la Universidad Nacional de Rio Cuarto. La experiencia desde la voz de los protagonistas", en M. Panaia, M. (coord.), Abandonar la universidad con o sin título, Buenos Aires, Miño y Dávila, pp. 81-108.

Cruz Ordiérez, D. e I. Ortega Calpa (2008), "Análisis de la deserción estudiantil en la facultad de ciencias exactas y naturales de la Universidad de Nariño desde la cohorte 2001-2 hasta la cohorte 2006-7 utilizando el sistema Spadies", San Juan Pasto, Colombia, Universidad de Nariño, disponible en: <http:// es.scribd.com/doc/243395183/ayuda-investigacionpdf> [consulta: 7 de Abril de 2014].

De Vries, W., P. León Arenas, J. F. Romero Muñoz e I. Hernández Saldaña (2011), “¿Desertores o decepcionados? Distintas causas para abandonar los estudios universitarios", en Revista de la Educación Superior, 40 (4), 29-50.
Díaz Peralta, G. (2008), "Modelo conceptual para la deserción estudiantil universitaria chilena”, en Estudios pedagógicos, 34 (2), pp. 65-86.

Domínguez, G. (2007), "Permanencia, graduación y deserción de estudiantes universitarios. Estudio de caso (1986-1999)", en 40 Congreso Nacional y 20 internacional de Investigación Educativa, Comahue [T 188. A. 2 CD ROM].

Elbaum, J. (1998), La escuela desde afuera. Culturas juveniles y abandono escolar. Propuesta educativa, Buenos Aires, FLACSO.

Fernández de Pinedo, I. (1982), “Construcción de una escala de actitudes tipo Likert”, Madrid, Ministerio de Trabajo y asuntos Sociales de España, Instituto Nacional de Seguridad e Higiene del Trabajo.

Follari, R. (2005), "El salto de la secundaria a la facultad aumenta la deserción universitaria", en Diario La Capital, 28 de mayo, p. 18.

Fontalvo Cerpa, W., M. Castillo González y S. Polo Cantillo (2014), "Análisis comparativo entre las características más relevantes de deserción estudiantil en el programa de Ingeniería Industrial de la Universidad Autónoma del Caribe. Estudiantes activos en el periodo 2013-01 y desertores académicos de los periodos 201101 a 2012-02", en Escenarios, 12(1), 96-104.

García de Fanelli, A. M. (2005), "Acceso, abandono y graduación en la educación superior argentina", en Sistema de Información de Tendencias Educativas en América Latina, disponible en: <http//www.sireal.lipe-oei.org>. Jewsbury, A. e I. Haefeli (2000), "Análisis de la deserción en universidades públicas argentinas", en V Congreso Internacional del CLAD, Santo Domingo, GLAD.

Lewis, Ann (2000), Convivencia infantil y discapacidad, México, Trillas.

Lorenzano, G. (2004), "La deserción universitaria en la Universidad Nacional de Tres de Febrero", Tesis de grado, Argentina, Universidad Nacional de Tres de Febrero.

Maya, M. V. (2013), "Investigación sobre la carrera 
de Licenciatura en Ciencias Biológicas, Facultad de Ciencias Naturales e IML (UNT). Análisis de ingresantes y egresados", Comunicación Personal.

Montes, H. (2002), "La transición de la educación media a la educación superior, Retención y movilidad estudiantil en la educación superior: calidad en la educación", Santiago de Chile, Publicación del Consejo Superior de Educación.

Nicoletti, V. R. (2010), "Acceso y permanencia del estudiante en la Universidad Argentina", Calidad de vida-Universidad de Flores, 1(5), 3-14, disponible en: $<$ http://www.calidaddevidauflo.com.ar> [consulta: 12 de Mayo de 2014].

Nunnally, J. C. (1967), "Alfa de Cronbach y consistencia interna de los ítems de un instrumento de medida", disponible en: <https://www.uv.es/ friasnav/ AlfaCronbach.pdf>

Panaia, M. (2013), "Abandonar la universidad: ¿decisión premeditada o imprevista?", en M. Panaia (comp.), Abandonar la universidad con o sin título, Buenos Aires: Miño y Dávila, pp. 21-65.

Ramallo, J. M. (2002), "Etapas históricas de la educación argentina. Novena Etapa: transformación del sistema", Buenos Aires, Fundación Nuestra Historia, disponible en: <http://www.argentinahistorica.com.ar/> [consulta: 13 de marzo de 2014].

Rizzuto, F. (2009), "La deserción en la educación superior, motivos y medidas preventivas", tesis, Argentina, Universidad Austral, disponible en: < http: //www.web.austral.edu.ar/educacion/tesis> [consulta: 5 de septiembre de 2013].

Santos Sharpe, A. I. (2016), "Un análisis histórico del abordaje sobre el abandono universitario en Argentina", en Anuario de Historia de la Educación, 17 (2), pp. 3-31.

Santos Sharpe, A.I. y S. Carli (2016), "Estudios globales y locales sobre el abandono de los estudios universitarios. Teorías, perspectivas y nuevos abordajes", en Revista Argentina de Educación Superior, 13, pp. 6-31.

Simone, V., I. Iavorski Losada y D. Wejchenberg (2013), "La construcción de población de abandonadores", en M. Panaia (comp.), en Abandonar la universidad con o sin título, Buenos Aires, Miño y Dávila, pp. 67-80.

Tinto, V. (2006-2007), "Research and practice of student retention: what next?", en College Student Retention, 8 (1), pp. 1-19.

Tinto, V. (1982), "Definir la deserción: una cuestión de perspectiva", en Revista de Educación Superior, núm. 71, disponible en: <http://www.publicaciones.anuies.mx/ revista/71> [consulta: 27 de febrero de 2014].

Troncoso, G. y S. Ávila. (2007), "Reflexiones sobre la deserción en la Universidad Nacional del Comahue", en IV Congreso Nacional y II Internacional de Investigación Educativa, Comahue (T.462. A.2 CD ROM).

Vélez, A. y D. F. López Jiménez (2004), "Estrategias para vencer la deserción universitaria", en Educación y Educadores, México, Universidad de La Sabana, pp. 177-203.

\section{Cómo citar este artículo:}

Maya, Mariela, Rosana González, Gabriela Aguirre, Jorgelina Juárez, Yanina Fernández y Virginia Abdala (2019), "El abandono en las carreras en Ciencias Biológicas de la Universidad Nacional de Tucumán. Década 1991-2001”, en Revista Iberoamericana de Educación Superior (RIES), México, UNAM-IISUE/Universia, vol. X, Núm. 27, pp. 131-156, DOI: https://doi.org/10.22201/iisue.20072872e.2019.28.43 3[consulta: fecha de última consulta]. 
Anexo 1. Registro de inscripciones y calificaciones

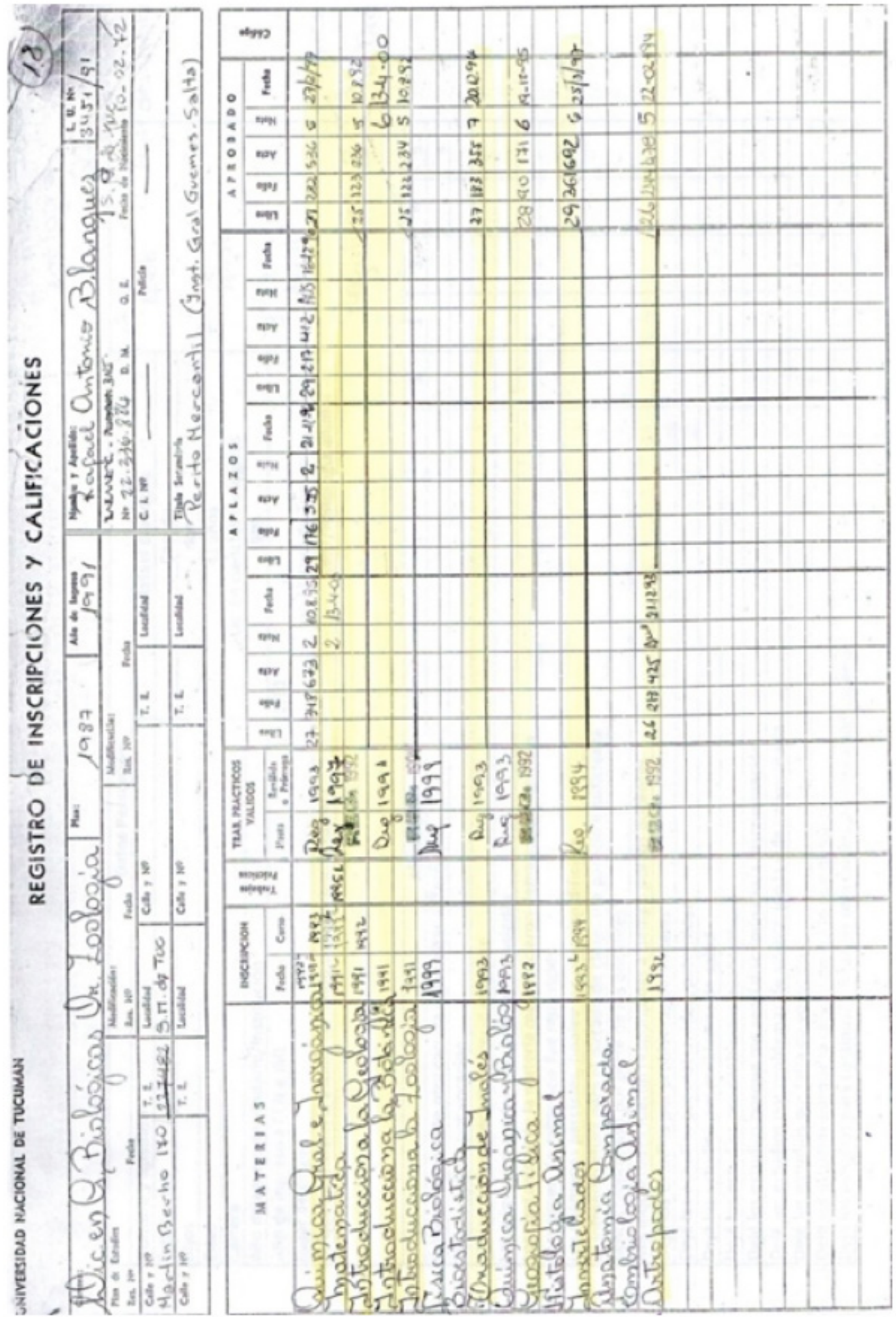


Anexo 2. Listado de materias por plan de estudio de cada carrera

\begin{tabular}{|c|c|c|c|}
\hline $\begin{array}{c}\text { Licenciatura en Ciencias } \\
\text { Biológicas Orientación Botánica }\end{array}$ & $\begin{array}{l}\text { Licenciatura en Ciencias } \\
\text { Biológicas Orientación Zoología }\end{array}$ & $\begin{array}{c}\text { Profesorado en Ciencias } \\
\text { Biológicas }\end{array}$ & $\begin{array}{c}\text { Profesorado en Ciencias } \\
\text { Naturales }\end{array}$ \\
\hline Química General e Inorgánica & Química General e Inorgánica & Introducción a la educación & Introducción a la Geología \\
\hline Matemáticas & Matemáticas & Introducción a la Geología & Introducción a la Botánica \\
\hline Introducción a la Geología & Introducción a la Geología & Física Biológica & Introducción a la Zoología \\
\hline Introducción a la Botánica & Introducción a la Botánica & Química general e Inorgánica & Química general e Inorgánica \\
\hline Introducción a la Zoología & Introducción a la Zoología & Biología general y Metodología de & Química Orgánica y Biológica \\
\hline Física Biológica & Física Biológica & las Ciencias & |nvertebrados | \\
\hline Bioestadística & Bioestadística & Psicología de la Educación & Fanerógamas \\
\hline Traducción de Inglés & Traducción de Inglés & Diversidad Animal I & Antropología \\
\hline Química Orgánica y Biológica & Química Orgánica y Biológica & Diversidad Vegetal I & Vertebrados \\
\hline Geografía Física & Geografía Física & Química Orgánica y Biológica & Criptógamas \\
\hline Fanerógamas I & Histología Animal & Didáctıca General & Invertebrados II (Artrópodos) \\
\hline Criptogramas I & Invertebrados & $\begin{array}{l}\text { Didáctica Especial de las Ciencias } \\
\text { Biológicas }\end{array}$ & Biogeografía \\
\hline Fanerógamas II & Anatomía Comparada & Citología y Genética & Anatomía Humana \\
\hline Criptogramas II & Embriología Animal & & Mineralogía y Petrografía \\
\hline Edafología & Artrópodos & & Fisiología Humana \\
\hline Anatomía Vegetal & Vertebrados & & Higiene y Puericultura \\
\hline Citología y Genética & Paleozoología I & & Citología y Genética \\
\hline Paleobotánica & Citología y Genética & & Lengua castellana \\
\hline Fisiología vegetal & Fisiología Animal & & |nglés | \\
\hline Ecología & Paleozoología II & & |nglés || \\
\hline \multirow[t]{6}{*}{ Fitogeografía } & Ecología & & Pedagogía \\
\hline & Biología Legal & & Psicología \\
\hline & Zoogeografía & & Didáctica General \\
\hline & & & Didáctica Especial y Práctica \\
\hline & & & Psicología de la EM \\
\hline & & & $\begin{array}{l}\text { Didáctica General y Organización } \\
\text { Escolar }\end{array}$ \\
\hline
\end{tabular}


Anexo 3. Índice de abandono

\begin{tabular}{|c|c|c|c|c|}
\hline Año & $N^{\circ}$ ingresantes & $N^{\circ}$ reinscritos & No abandonan & Frecuencias de abandono \\
\hline 1990 & $\begin{array}{l}\text { B-38 } \\
\text { Z-62 } \\
\text { LIC: } 113 \\
\text { PROF: } 21\end{array}$ & $\begin{array}{l}\text { B-50 } \\
\text { Z-68 } \\
\text { LIC:100 } \\
\text { PROF: } 21\end{array}$ & $x$ & $x$ \\
\hline 1991 & $\begin{array}{l}\text { B-35 } \\
\text { Z-78 } \\
\text { LIC: } 113 \\
\text { PROF: } 28\end{array}$ & $\begin{array}{l}\text { B-57 } \\
\text { Z-139 } \\
\text { LIC:196 } \\
\text { PROF:27 }\end{array}$ & $\begin{array}{l}\text { B-31 } \\
\text { Z-9 } \\
\text { LIC:40 } \\
\text { PROF: }\end{array}$ & $\begin{array}{l}\text { B-18 } \\
Z-47 \\
\text { LIC:65 } \\
\text { PROF:1 }\end{array}$ \\
\hline 1992 & $\begin{array}{l}\text { B-33 } \\
\text { Z-82 } \\
\text { LIC:115 } \\
\text { PROF:21 }\end{array}$ & $\begin{array}{l}\text { B-66 } \\
\text { Z-153 } \\
\text { LIC:219 } \\
\text { PROF:44 }\end{array}$ & $\begin{array}{l}\text { B-26 } \\
Z-64 \\
\text { LIC:90 } \\
\text { PROF:11 }\end{array}$ & $\begin{array}{l}\text { B-25 } \\
Z-51 \\
\text { LIC:76 } \\
\text { PROF:23 }\end{array}$ \\
\hline 1993 & $\begin{array}{l}\text { B-36 } \\
\text { Z-163 } \\
\text { LIC:199 } \\
\text { PROF:43 }\end{array}$ & $\begin{array}{l}\text { B-49 } \\
\text { Z-154 } \\
\text { LIC: } 203 \\
\text { PROF:42 }\end{array}$ & $\begin{array}{l}\text { B-50 } \\
\text { Z-81 } \\
\text { LIC:121 } \\
\text { PROF:23 }\end{array}$ & $\begin{array}{l}\text { B-9 } \\
Z-0 \\
\text { LIC:9 } \\
\text { PROF:1 }\end{array}$ \\
\hline 1994 & $\begin{array}{l}\text { B-17 } \\
\text { Z VET-136 } \\
\text { Z-96 } \\
\text { LIC:250 } \\
\text { PROF:60 }\end{array}$ & $\begin{array}{l}\text { B-44 } \\
\text { Z-196 } \\
\text { LIC:240 } \\
\text { PROF: }\end{array}$ & $\begin{array}{l}\text { B-41 } \\
\text { Z-121 } \\
\text { LIC:162 } \\
\text { PROF: }\end{array}$ & $\begin{array}{l}\text { B-25 } \\
\text { Z-30 } \\
\text { LIC:55 } \\
\text { PROF: }\end{array}$ \\
\hline 1995 & $\begin{array}{l}\text { B-32 } \\
\text { Z-151 } \\
\text { LIC:183 } \\
\text { PROF:69 }\end{array}$ & $\begin{array}{l}\text { B- } 39 \\
\text { Z-195 } \\
\text { LIC:234 } \\
\text { PROF:56 }\end{array}$ & $\begin{array}{l}\text { B- } 22 \\
\text { Z-233 } \\
\text { LIC:255 } \\
\text { PROF: }\end{array}$ & $\begin{array}{l}\text { B-7 } \\
Z-44 \\
\text { LIC:51 } \\
\text { PROF:13 }\end{array}$ \\
\hline 1996 & $\begin{array}{l}\text { B- } 39 \\
\text { Z-154 } \\
\text { LIC:193 } \\
\text { PROF:103 }\end{array}$ & $\begin{array}{l}\text { B-51 } \\
\text { Z-211 } \\
\text { LIC:262 } \\
\text { PROF:84 }\end{array}$ & $\begin{array}{l}\text { B- } 20 \\
\text { Z- } 135 \\
\text { LIC: } 155 \\
\text { PROF: } 41\end{array}$ & $\begin{array}{l}\text { B-12 } \\
Z-57 \\
\text { LIC:69 } \\
\text { PROF:19 }\end{array}$ \\
\hline 1997 & $\begin{array}{l}\text { B-21 } \\
\text { Z-125 } \\
\text { LIC:146 } \\
\text { PROF:103 }\end{array}$ & $\begin{array}{l}\text { B-59 } \\
\text { Z-238 } \\
\text { LIC:297 } \\
\text { PROF:103 }\end{array}$ & $\begin{array}{l}\text { B-31 } \\
\text { Z-127 } \\
\text { LIC:158 } \\
\text { PROF:75 }\end{array}$ & $\begin{array}{l}\text { B- } 38 \\
\text { Z-109 } \\
\text { LIC:147 } \\
\text { PROF:0 }\end{array}$ \\
\hline
\end{tabular}

Botánica (B), Zoología (Z), Licenciatura (LIC), Profesorado (PROF). 
Anexo 3. Análisis de cantidad de respuestas por ítem

\begin{tabular}{|c|c|c|c|c|c|}
\hline Núm. de ítem & Opción 1 & Opción 2 & Opción 3 & Opción 4 & Opción 5 \\
\hline Ítem 1 & 9 & 0 & 0 & 0 & 13 \\
\hline Ítem 2 & 15 & 2 & 2 & 0 & 3 \\
\hline Ítem 3 & 2 & 2 & 10 & 3 & 5 \\
\hline Ítem 4 & 3 & 1 & 5 & 4 & 6 \\
\hline Ítem 5 & 3 & 2 & 7 & 3 & 7 \\
\hline Ítem 6 & 3 & 3 & 3 & 2 & 11 \\
\hline Ítem 7 & 4 & 1 & 7 & 4 & 6 \\
\hline Ítem 8 & 4 & 0 & 5 & 4 & 9 \\
\hline Ítem 9 & 6 & 4 & 7 & 4 & 1 \\
\hline Ítem 10 & 7 & 4 & 5 & 4 & 2 \\
\hline Ítem 11 & 16 & 0 & 0 & 1 & 5 \\
\hline Ítem 12 & 7 & 1 & 0 & 0 & 14 \\
\hline Ítem 13 & 3 & 0 & 0 & 0 & 19 \\
\hline Ítem 14 & 2 & 1 & 1 & 1 & 17 \\
\hline Ítem 15 & 0 & 1 & 0 & 0 & 21 \\
\hline Ítem 16 & 1 & 0 & 1 & 1 & 18 \\
\hline Ítem 17 & 0 & 1 & 0 & 0 & 21 \\
\hline Ítem 18 & 1 & 1 & 3 & 1 & 15 \\
\hline Îtem 19 & 8 & 0 & 0 & 0 & 14 \\
\hline
\end{tabular}


Anexo 4. Valoración escala y resultados de la escala Likert

\begin{tabular}{|c|c|c|c|c|c|c|}
\hline Núm. de ítem & $\begin{array}{l}\text { Muy en } \\
\text { acuerdo }\end{array}$ & De acuerdo & Neutro & $\begin{array}{c}\text { En } \\
\text { desacuerdo }\end{array}$ & $\begin{array}{c}\text { En total } \\
\text { desacuerdo }\end{array}$ & Pregunta \\
\hline 1 & 5 & 4 & 3 & 2 & 1 & positiva \\
\hline 2 & 5 & 4 & 3 & 2 & 1 & positiva \\
\hline 3 & 1 & 2 & 3 & 4 & 5 & negativa \\
\hline 4 & 1 & 2 & 3 & 4 & 5 & negativa \\
\hline 5 & 1 & 2 & 3 & 4 & 5 & negativa \\
\hline 6 & 1 & 2 & 3 & 4 & 5 & negativa \\
\hline 7 & 1 & 2 & 3 & 4 & 5 & negativa \\
\hline 8 & 1 & 2 & 3 & 4 & 5 & negativa \\
\hline 9 & 5 & 4 & 3 & 2 & 1 & positiva \\
\hline 10 & 5 & 4 & 3 & 2 & 1 & positiva \\
\hline 11 & 1 & 2 & 3 & 4 & 5 & negativa \\
\hline 12 & 1 & 2 & 3 & 4 & 5 & negativa \\
\hline 13 & 1 & 2 & 3 & 4 & 5 & negativa \\
\hline 14 & 1 & 2 & 3 & 4 & 5 & negativa \\
\hline 15 & 1 & 2 & 3 & 4 & 5 & negativa \\
\hline 16 & 1 & 2 & 3 & 4 & 5 & negativa \\
\hline 17 & 1 & 2 & 3 & 4 & 5 & negativa \\
\hline 18 & 1 & 2 & 3 & 4 & 5 & negativa \\
\hline 19 & 1 & 2 & 3 & 4 & 5 & negativa \\
\hline
\end{tabular}




\begin{tabular}{|c|c|c|c|c|c|c|c|c|c|c|c|c|c|c|c|c|c|c|c|c|c|c|c|}
\hline 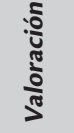 & 偦 & $\begin{array}{l}\text { 童 } \\
\text { 鈿 }\end{array}$ & 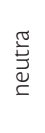 & $\begin{array}{l}\text { 离 } \\
\text { 总 } \\
0\end{array}$ & 竞 & & 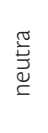 & 谞 & 雚 & 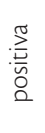 & 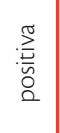 & 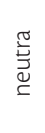 & 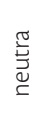 & 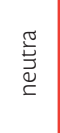 & 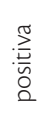 & $\begin{array}{l}\text { 童 } \\
\text { 鈿 }\end{array}$ & 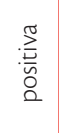 & 结 & 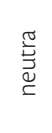 & 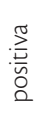 & $\stackrel{\frac{\pi}{5}}{\underline{\underline{E}}}$ & 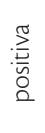 & \\
\hline 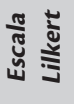 & $\checkmark$ & $\checkmark$ & $m$ & $\checkmark$ & $\checkmark$ & $m$ & $m$ & $\checkmark$ & $\checkmark$ & $\checkmark$ & $\checkmark$ & $m$ & $m$ & $m$ & $\checkmark$ & $\checkmark$ & $\checkmark$ & $\checkmark$ & $m$ & $\checkmark$ & $m$ & $\checkmark$ & \\
\hline 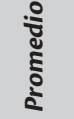 & $\mathcal{f}$ & $\bar{\checkmark}$ & $\bar{m}$ & $\bar{f}$ & $\stackrel{\circ}{\forall}$ & $\stackrel{m}{m}$ & $\bar{m}$ & $\stackrel{\infty}{m}$ & & $\underset{\forall}{\xi}$ & $\underset{f}{\sim}$ & $\stackrel{m}{m}$ & $\stackrel{m}{m}$ & $\stackrel{m}{m}$ & $\stackrel{\circ}{\text { m }}$ & $\stackrel{m}{m}$ & $\underset{\forall}{f}$ & $\vec{m}$ & $\overrightarrow{\mathrm{N}}$ & $\check{\forall}$ & $\bar{m}$ & $\vec{m}$ & \\
\hline స్ّ & $\bar{\infty}$ & $\stackrel{\infty}{\wedge}$ & in & $\stackrel{\infty}{\wedge}$ & 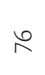 & $\hat{\sigma}$ & $\stackrel{\infty}{n}$ & $\stackrel{n}{n}$ & $\curvearrowright$ & $\infty$ & $\infty$ & ఫ & $\tilde{\sigma}$ & ¿̛ & ळ & $N$ & $\infty$ & $\stackrel{ \pm}{\wedge}$ & $\stackrel{\circ}{n}$ & $\stackrel{\infty}{\curvearrowright}$ & 8 & $\stackrel{n}{\wedge}$ & 0 \\
\hline 2 & - & - & in & $r$ & n & - & - & in & n & in & in & - & in & $n$ & in & - & $n$ & $n$ & in & - & in & - & 0 \\
\hline$\stackrel{\infty}{\sim}$ & in & $\checkmark$ & in & in & in & in & $m$ & in & in & in & in & $m$ & in & - & $\sim$ & $m$ & $n$ & in & in & in & in & in & 0 \\
\hline$\approx$ & in & in & in & in & in & in & in & in & in & in & in & in & in & in & $\sim$ & in & in & in & in & in & in & in & 0 \\
\hline$\stackrel{2}{2}$ & $\checkmark$ & n & in & $\checkmark$ & in & - & in & in & in & in & in & in & in & in & $m$ & in & $n$ & in & in & in & in & in & 0 \\
\hline$\underline{n}$ & in & in & in & in & in & in & $\sim$ & in & in & in & in & in & in & in & in & in & in & in & in & in & in & in & 0 \\
\hline \pm & in & in & in & in & in & $\sim$ & - & in & in & in & in & in & in & in & in & in & in & in & - & in & - & in & 0 \\
\hline$m$ & in & in & in & in & in & $\checkmark$ & - & in & - & in & in & in & in & in & in & in & in & in & - & in & in & in & 0 \\
\hline$\approx$ & in & in & - & - & in & in & - & - & in & - & in & in & - & in & in & in & $n$ & in & $\sim$ & in & - & in & 0 \\
\hline$=$ & in & - & $r$ & in & - & - & in & - & in & - & - & - & $r$ & in & $\checkmark$ & in & - & - & - & - & - & in & 0 \\
\hline$\therefore$ & in & $m$ & $\sim$ & $\checkmark$ & $\checkmark$ & $m$ & $m$ & $\sim$ & $m$ & in & - & $\sim$ & in & - & $\sim$ & $\sim$ & in & $\checkmark$ & in & in & $m$ & - & 0 \\
\hline$a$ & $\checkmark$ & $m$ & $m$ & in & $\checkmark$ & $m$ & $m$ & $\sim$ & $m$ & $\checkmark$ & in & $\sim$ & in & $m$ & $\sim$ & $\sim$ & $\triangleleft$ & in & in & in & $m$ & - & 0 \\
\hline$\infty$ & $m$ & in & in & in & - & in & $m$ & in & in & in & in & $\checkmark$ & - & $m$ & in & $\checkmark$ & $\checkmark$ & $n$ & - & $m$ & - & in & 0 \\
\hline$n$ & $\checkmark$ & in & $\sim$ & in & $\checkmark$ & $m$ & $m$ & $\checkmark$ & $m$ & n & $m$ & $m$ & - & - & in & $\checkmark$ & $m$ & in & - & $\checkmark$ & $m$ & in & 0 \\
\hline 6 & in & in & - & $\checkmark$ & in & in & $m$ & in & in & in & $m$ & $m$ & - & $\sim$ & in & $m$ & in & $\sim$ & - & in & $\sim$ & in & 0 \\
\hline in & in & in & - & in & $\checkmark$ & $m$ & $m$ & $\checkmark$ & in & in & $\checkmark$ & $m$ & - & $\sim$ & $m$ & $\checkmark$ & $m$ & $\sim$ & - & in & $m$ & in & 0 \\
\hline$\checkmark$ & in & in & - & in & $\checkmark$ & $m$ & $m$ & $\checkmark$ & in & in & in & $m$ & - & $m$ & $m$ & $\checkmark$ & $\checkmark$ & $\sim$ & - & in & $m$ & in & 0 \\
\hline$m$ & $\checkmark$ & in & - & in & $m$ & $m$ & $m$ & $\checkmark$ & $m$ & in & $m$ & $m$ & in & $m$ & $\sim$ & $\checkmark$ & $m$ & $\sim$ & - & $m$ & $m$ & in & 0 \\
\hline$N$ & in & in & in & $m$ & in & in & in & in & in & in & in & in & - & $\checkmark$ & $\checkmark$ & in & $n$ & in & in & in & in & - & 0 \\
\hline- & - & - & - & - & - & - & in & - & - & - & in & - & in & - & - & - & $n$ & - & in & - & - & - & 0 \\
\hline * & - & N & $m$ & $\checkmark$ & in & 0 & $r$ & $\infty$ & $a$ & $\stackrel{\circ}{\circ}$ & $=$ & $\simeq$ & $\stackrel{m}{=}$ & \pm & 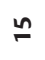 & $\stackrel{\circ}{\circ}$ & $=$ & $\stackrel{\infty}{\sim}$ & $\stackrel{9}{ }$ & $\stackrel{\text { N }}{\text { s. }}$ & $\bar{\sim}$ & $\tilde{N}$ & $\stackrel{\sim}{\sim}$ \\
\hline
\end{tabular}


Anexo 5. Año de mayor abandono

\begin{tabular}{|c|c|c|c|}
\hline Encuestado & $\begin{array}{c}\text { Año de cursado } \\
\text { en el que } \\
\text { abandona }\end{array}$ & Año de ingreso & Carrera \\
\hline 1 & $3^{\circ}$ & 1990 & Licenciatura \\
\hline 2 & $2^{\circ}$ & 1990 & Licenciatura \\
\hline 3 & $4^{\circ}$ & 1996 & Profesorado \\
\hline 4 & $2^{\circ}$ & 1996 & Profesorado \\
\hline 5 & $3^{\circ}$ & 1990 & Licenciatura \\
\hline 6 & $3^{\circ}$ & 1991 & Licenciatura \\
\hline 7 & $3^{\circ}$ & 1992 & Profesorado \\
\hline 8 & $3^{\circ}$ & 1990 & Licenciatura \\
\hline 9 & $3^{\circ}$ & 1990 & Profesorado \\
\hline 10 & $4^{\circ}$ & 1991 & Licenciatura \\
\hline 11 & $2^{\circ}$ & 1996 & Licenciatura \\
\hline 12 & $2^{\circ}$ & 1990 & Profesorado \\
\hline 13 & $3^{\circ}$ & 1993 & Licenciatura \\
\hline 14 & $3^{\circ}$ & 1990 & Profesorado \\
\hline 15 & $3^{\circ}$ & 1991 & Licenciatura \\
\hline 16 & $2^{\circ}$ & 1991 & Licenciatura \\
\hline 17 & $3^{\circ}$ & 1991 & Licenciatura \\
\hline 18 & $3^{\circ}$ & 1991 & Licenciatura \\
\hline 19 & $4^{\circ}$ & 1996 & Profesorado \\
\hline 20 & $1^{\circ}$ & 1996 & Profesorado \\
\hline 21 & $3^{\circ}$ & 1994 & Licenciatura \\
\hline 22 & $1^{\circ}$ & 1990 & Licenciatura \\
\hline
\end{tabular}

